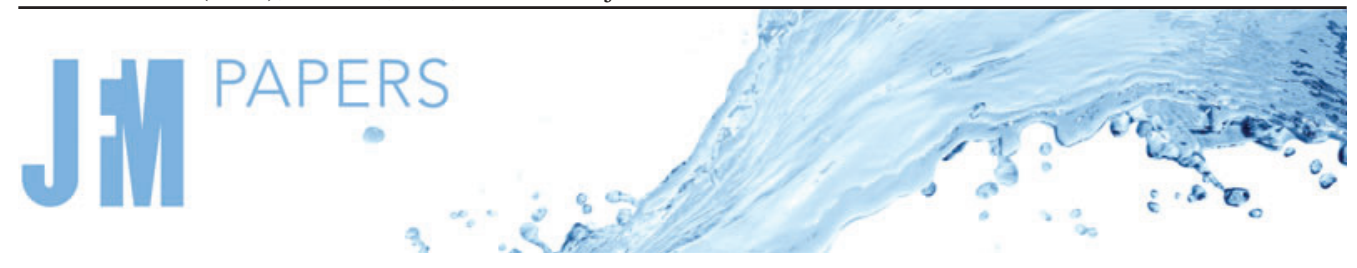

\title{
Asymptotics of streamwise Reynolds stress in wall turbulence
}

\author{
Peter A. Monkewitz $\dagger$ \\ École Polytechnique Fédérale de Lausanne (EPFL), CH-1015 Lausanne, Switzerland
}

(Received 14 April 2021; revised 29 September 2021; accepted 18 October 2021)

The scaling of different features of streamwise normal stress profiles $\langle u u\rangle^{+}\left(y^{+}\right)$in turbulent wall-bounded flows is the subject of a long-running debate. Particular points of contention are the scaling of the 'inner' and 'outer' peaks of $\langle u u\rangle^{+}$at $y^{+} \approx 15$ and $y^{+}=O\left(10^{3}\right)$, respectively, their infinite Reynolds number limit, and the rate of logarithmic decay in the outer part of the flow. Inspired by the thought-provoking paper of Chen \& Sreenivasan (J. Fluid Mech., vol. 908, 2021, p. R3), two terms of an inner asymptotic expansion of $\langle u u\rangle^{+}$in the small parameter $\operatorname{Re}_{\tau}^{-1 / 4}$ are constructed from a set of direct numerical simulations (DNS) of channel flow. This inner expansion is for the first time matched through an overlap layer to an outer expansion, which not only fits the same set of channel DNS within $1.5 \%$ of the peak stress, but also provides a good match of laboratory data in pipes and the near-wall part of boundary layers, up to the highest $R e_{\tau}$ values of $10^{5}$. The salient features of the new composite expansion are first, an inner $\langle u u\rangle^{+}$peak, which saturates at 11.3 and decreases as $R e_{\tau}^{-1 / 4}$. This inner peak is followed by a short 'wall log law' with a slope that becomes positive for $R e_{\tau}$ beyond $O\left(10^{4}\right)$, leading up to an outer peak, followed by the logarithmic overlap layer with a negative slope going continuously to zero for $R e_{\tau} \rightarrow \infty$.

Key words: turbulent boundary layers

\section{Introduction}

In the following, the classical non-dimensionalization is adopted with the friction velocity $\widehat{u}_{\tau} \equiv\left(\widehat{\tau}_{w} / \widehat{\rho}\right)^{1 / 2}$ and the 'inner' or viscous length scale $\widehat{\ell} \equiv\left(\widehat{v} / \widehat{u}_{\tau}\right)$, where $\widehat{\tau_{w}}, \widehat{\rho}$ and $\widehat{v}$ are the wall shear stress, density and dynamic viscosity, respectively, and hats identify dimensional quantities. The resulting non-dimensional inner and outer wall-normal coordinates are $y^{+}=\hat{y} / \widehat{\ell}$ and $Y=y^{+} / R e_{\tau}$, respectively, with $R e_{\tau} \equiv \hat{L} / \widehat{\ell}$ the friction

$\dagger$ Email address for correspondence: peter.monkewitz@epfl.ch 


\section{P.A. Monkewitz}

Reynolds number and $\hat{L}$ the outer length scale such as channel half-height, pipe radius or appropriately chosen boundary layer thickness in unconfined turbulent wall layers.

The scaling of normal Reynolds stresses in turbulent boundary layers, in particular of the streamwise component $\langle u u\rangle^{+}$, which is experimentally accessible with single hot-wires, has been and remains a subject of vigorous debate. Research in this area involves, in different proportions, data analysis and the correlation of profile characteristics with dominant balances in the governing equations or with simplified models of typical turbulent structures. Any complete summary of previous works on the subject goes beyond a research paper and the reader is referred to the review articles by Marusic et al. (2010) and Smits, McKeon \& Marusic (2011) for a general introduction.

The specific topic of the present paper is the construction, believed to be the first, of a complete composite matched asymptotic expansion (abbreviated MAE, see e.g. Kevorkian \& Cole 1981; Wilcox 1995) for the streamwise Reynolds stress $\langle u u\rangle^{+}$from available channel flow direct numerical simulations (DNS) and, at higher $R e_{\tau}$, experimental pipe flow profiles. Unlike the MAE for the mean velocity profile (see e.g. Monkewitz 2021), which provides, at higher laboratory Reynolds numbers, an excellent approximation of $U^{+}$already at leading order, the MAE of the $\langle u u\rangle^{+}$profile requires at least two orders to properly describe its substantial evolution with $R e_{\tau}$.

One of the principal questions being currently debated is the scaling of the inner peak height $\langle u u\rangle_{I P}^{+}$located at $y^{+} \approx 15$. According to one school of thought, this inner-scaled peak grows indefinitely with $\ln R e_{\tau}$, according to Samie et al. (2018) as $3.54+0.646 \ln R e_{\tau}$ in the zero pressure gradient turbulent boundary layer (ZPG TBL). In order to obtain a finite non-dimensional inner peak in the limit of infinite $\operatorname{Re}_{\tau}$ with this scaling, $\langle\hat{u} \hat{u}\rangle$ would have to be normalized by the product of $\hat{u}_{\tau}$ and the outer velocity scale, as proposed by DeGraaff \& Eaton (2000), for instance. However, Chauhan, Monkewitz \& Nagib (2009) have shown that this 'mixed scaling' does not significantly improve the collapse of profiles at different $R e_{\tau}$. The logarithmic scaling of the inner peak has nevertheless gained widespread acceptance, because it corresponds to a key prediction of the attached eddy model, originally proposed by Townsend (1976), developed in Melbourne by Perry, Henbest \& Chong (1986), among others, and recently reviewed by Marusic \& Monty (2019). Closely linked to the unlimited growth of $\langle u u\rangle_{I P}^{+}$is the model prediction of a universal logarithmic decay, $\langle u u\rangle^{+}=1.95-1.26 \ln Y$ in the outer region (see e.g. Marusic et al. 2013).

Both of these model predictions have been challenged by Monkewitz \& Nagib (2015) (see also Monkewitz, Nagib \& Boulanger 2017), who have shown that, for the ZPG TBL, an unlimited growth of the inner peak $\langle u u\rangle_{I P}^{+}$is incompatible with the Taylor expansion of the full streamwise mean momentum equation about $y^{+}=0$. Recently, this view has received support from Chen \& Sreenivasan (2021), henceforth abbreviated CS2021, also for streamwise homogeneous flows such as channel and pipe flows. They argued, based on the maximum of $1 / 4$ for the turbulent energy dissipation rate, that the infinite Reynolds number limit of the inner peak height is finite and decreases from there as $\operatorname{Re}_{\tau}^{-1 / 4}$. It is noted, however, that these results (or any other scalings) have not yet been formally related to the Reynolds stress transport equations. This, and the limited variation of $\operatorname{Re}_{\tau}^{-1 / 4},\left(1 / \ln R e_{\tau}\right)$ and $\ln R e_{\tau}$ over the $R e_{\tau}$ range, where reliable streamwise normal stress data are available, go a long way to explaining the continuing disagreement on their scaling.

Leaving open questions for the concluding $\S 5$, the asymptotic sequence $\left\{1, \operatorname{Re}_{\tau}^{-1 / 4}, \ldots\right\}$ of CS2021 is adopted to construct, in $\S 2$, the two-term inner asymptotic expansion of 


$\begin{array}{lll}\text { Profile } & \operatorname{Re}_{\tau} & \text { Reference } \\ 1 & 5186 & \text { Lee \& Moser (2015) } \\ 2 & 3000 & \text { Thais, Mompean \& Gatski (2013) } \\ 3 & 2004 & \text { Hoyas \& Jiménez (2006) } \\ 4 & 1995 & \text { Lee \& Moser (2015) } \\ 5 & 1000 & \text { Lee \& Moser (2015) }\end{array}$

Table 1. Channel DNS profiles used to determine the first two terms of the asymptotic expansion (2.1).

the streamwise normal stress from channel flow DNS. Analytic fits for both terms of the near-wall asymptotic expansion are developed in Appendix A and continued to a logarithmic overlap layer. The matching outer expansion is developed in $\S 3$, resulting in the first complete two-term composite expansion of $\langle u u\rangle^{+}$, which successfully describes DNS and experimental $\langle u u\rangle^{+}$profiles for Reynolds numbers ranging from $10^{3}$ to $10^{5}$. Close to the wall, however, discrepancies, in particular of inner peak heights, exist between some measurements with the nano-scale thermal anemometry probe, known as NSTAP (Vallikivi \& Smits 2014), and the proposed composite profile. An explanation for these discrepancies is proposed in Appendix B.

The short $\S 4$ is devoted to a detailed comparison between outer peak heights and locations, obtained from the composite expansion, and available data. The concluding $\S 5$, finally, offers speculations on how the successful two-term expansion of $\langle u u\rangle^{+}$, based on the inner asymptotic sequence $\left\{1, \operatorname{Re}_{\tau}^{-1 / 4}, \ldots\right\}$ proposed by CS2021, could be related to the asymptotics of other terms in the transport equation for $\langle u u\rangle^{+}$. The section concludes with a list of further comments and observations. Some supplementary materials are available at https://doi.org/10.1017/jfm.2021.924, notably a comparison with the patched asymptotic expansion of Marusic \& Kunkel (2003) ('patched', because their inner and outer expansions are not matched in an overlap layer, but patched across a fixed $y^{+}$ interval).

\section{The inner asymptotic expansion of $\langle u u\rangle^{+}$and its inner peak}

The inner asymptotic expansion of the streamwise normal stress $\langle u u\rangle^{+}$for large $R e_{\tau}$ is extracted from the channel DNS of table 1 in a similar fashion as the mean velocity expansion in Monkewitz (2021), i.e. without recourse to a model.

Generalizing the Reynolds number dependence of the gauge function in (3.1) of Monkewitz (2021) from $R e_{\tau}^{-1}$ to $\Phi\left(R e_{\tau}\right)$, the first two terms of the asymptotic expansion

$$
\langle u u\rangle_{D N S}^{+}=f\left(y^{+}\right)+\Phi\left(R e_{\tau}\right) g\left(y^{+}\right),
$$

are obtained for various gauge functions $\Phi$ from pairs of DNS profiles at different $R e_{\tau}$. A good collapse of the $f$ and $g$ values from different profile pairs signifies that the gauge function $\Phi$ has been properly chosen and that higher-order terms in the expansion (2.1) are small or absent.

It turns out that $\Phi=\operatorname{Re}_{\tau}^{-1 / 4}$ and $\Phi=1 / \ln R e_{\tau}$ both produce a good collapse of the functions $f$ and $g$ obtained from all possible profile pairs in table 1, with $f\left(y^{+}\right)$the finite limit of $\langle u u\rangle^{+}$for $R e_{\tau} \rightarrow \infty$. The choice of $\Phi=R e_{\tau}^{-1 / 4}$ for the present paper, proposed by CS2021, is based on the following considerations: 


\section{P.A. Monkewitz}

(i) Arguments in favour of $\Phi=\operatorname{Re}_{\tau}^{-1 / 4}$ : the decomposition (2.1) with $\Phi=\operatorname{Re}_{\tau}^{-1 / 4}$ is shown in figure 1 and the collapse from different profile pairs on the fit (2.2) is seen to be rather good up to $y^{+}$of approximately 200. Furthermore, the leading term of the Taylor expansion (2.3) of (2.2) about the wall, shown in figure 1 as dotted lines, corresponds to an upper bound of $1 / 4$ for the dissipation rate at the wall, as argued by CS2021. They have furthermore argued, that the coefficient of $\left(y^{+}\right)^{2}$ in the Taylor expansion about the wall and the inner peak height $\langle u u\rangle_{I P}^{+}$are strictly proportional. Without having imposed this constraint on the fit (2.2), it yields a near perfect proportionality between (2.4) and the coefficient of $\left(y^{+}\right)^{2}$ in (2.3), the proportionality factor being $45\left(1+0.11 / R e_{\tau}\right)$. This proportionality has recently received strong support from the extensive data analyses of Hultmark \& Smits (2021) and Smits et al. (2021), who found a ratio of 46, independent of Reynolds number.

(ii) Arguments against $\Phi=1 / \ln R e_{\tau}$ : the decomposition (2.1) with $\Phi=1 / \ln R e_{\tau}$, on the other hand, is shown in figure 1 of the supplementary material and is seen to produce an equally good collapse of the $f$ and $g$ functions from different DNS pairs. The Taylor expansion of $\langle u u\rangle^{+}$about the wall, $\left(0.30-0.86 / \ln R e_{\tau}\right)\left(y^{+}\right)^{2}+\cdots$ is also in good agreement with Smits et al. (2021). However, as the coefficient of $\left(y^{+}\right)^{2}$ exceeds the limit of $1 / 4$ inferred by CS2021, this scaling is not pursued further.

(iii) Arguments against $\Phi=\ln R e_{\tau}$ : the choice of $\Phi=\ln R e_{\tau}$ in (2.1), finally, which corresponds to the inner peak scaling of the attached eddy model (Marusic \& Monty 2019), produces no comparable collapse of the $f$ and $g$ values from different DNS pairs of table 1, as seen in figure 2 of the supplementary material, and, of course, $\langle u u\rangle_{I P}^{+} \rightarrow \infty$ for $R e_{\tau} \rightarrow \infty$. For the simplest case of channel flow, the transport equation for $\langle u u\rangle^{+}$(see for instance Hinze (1975), (4) and (5)) is in all likelihood unbalanced with this scaling: based on the available profiles, notably those of Lee \& Moser (2015) at $R e_{\tau}=5186$, the viscous transport term $\mathcal{D}^{+}=$ $(1 / 2)\left[\mathrm{d}^{2}\langle u u\rangle^{+} /\left(\mathrm{d} y^{+}\right)^{2}\right]$ becomes negative beyond $y^{+} \approx 5$. In the neighbourhood of $y_{I P}^{+} \approx 15$, it may be approximated by $-\langle u u\rangle_{I P}^{+}\left(y_{I P}^{+}\right)^{-2}$. Hence, with $\langle u u\rangle_{I P}^{+}$scaling as $\ln R e_{\tau}$, the viscous transport term in the neighbourhood of the inner peak goes to negative infinity for $R e_{\tau} \rightarrow \infty$. For the DNS of Lee \& Moser (2015) at $R e_{\tau}=5186$, all the other terms of the transport equation are negative around $y_{I P}^{+}$, except the production term, which is limited to $1 / 4$ (see e.g. Pope (2000), exercise 7.6). With the reasonable assumption that, between $R e_{\tau}=5186$ and infinity, no term of the transport equation for $\langle u u\rangle^{+}$becomes positive unbounded in a neighbourhood of $y_{I P}^{+}$, the equation is clearly asymptotically unbalanced. The choice of $\Phi=\ln R e_{\tau}$ is therefore abandoned at this point.

Proceeding with the asymptotic sequence $\left\{1, \operatorname{Re}_{\tau}^{-1 / 4}, \ldots\right\}$, the functions $f$ and $g$ of figure 1 need to be fitted in order to generate $\langle u u\rangle^{+}$profiles for any Reynolds number. Two features of $f$ and $g$ will prove to be important for the construction of the complete inner asymptotic expansion: the expected 'hump' at $y^{+} \approx 15$, and the rather clear logarithmic region between $y^{+} \approx 60$ and 200 , with a logarithmic slope that decreases as $\operatorname{Re}_{\tau}^{-1 / 4}$ from its maximum of 0.85 at infinite $\operatorname{Re}_{\tau}$ (figure $1 a$ ). For the $O(1)$ part $f$, the fit $\mathcal{M}_{2}$ (A1), constructed from a Padé approximant for the derivative, analogous to the construction of the Musker mean velocity profile (Musker 1979), has been developed and is supplemented by the 'hump' function $\mathcal{H}(\mathrm{A} 4)$. The $O\left(\operatorname{Re}_{\tau}^{-1 / 4}\right)$ part $g$ is well fitted by the 'corner function' $\mathcal{C}$ (A5) plus a 'hump.' Hence, the near-wall stress, up to higher-order terms (HOT), is 


\section{Streamwise Reynolds stress}
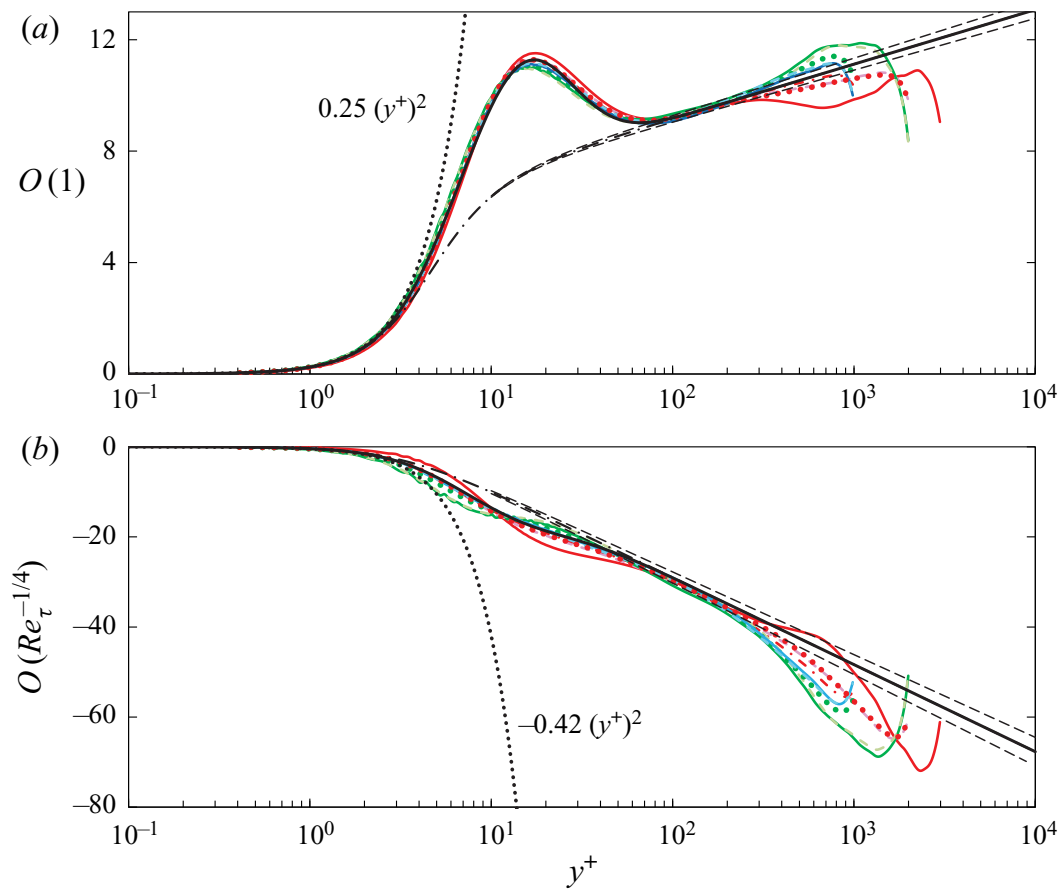

Figure 1. The $O(1)(a)$ and $O\left(1 / R_{\tau}^{1 / 4}\right)(b)$ components of $\langle u u\rangle^{+}$extracted from DNS pairs of table 1: (red) profiles 1 and $2(-), 1$ and $3(--), 1$ and $4(\cdots), 1$ and $5(---)$; (green) 2 and $3(-), 2$ and $4(---), 2$ and $5(\cdots)$; (blue) 3 and 5 (-), 4 and $5(--)$. Panel (a) (black) - and - - -, O(1) part of (2.2) with and without hump. Panel (b) (black) - and - - - $O\left(1 / R e_{\tau}^{1 / 4}\right)$ part with and without hump; (black) - - -, logarithmic slopes modified by $\pm 5 \%$.

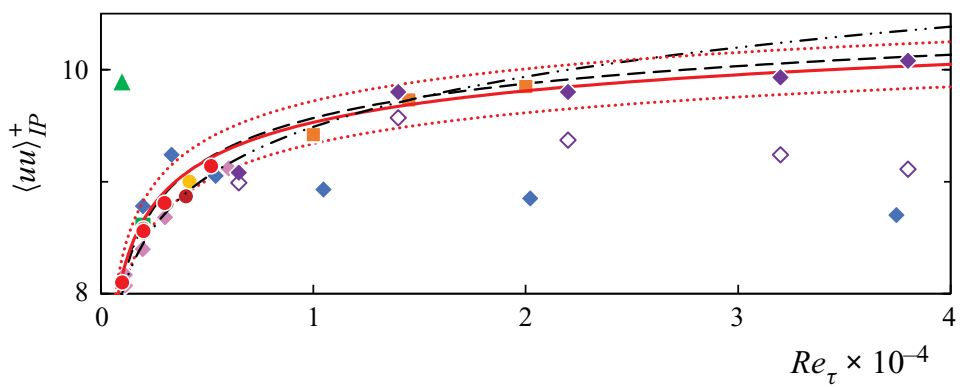

Figure 2. Inner peak height $\langle u u\rangle_{I P}^{+}$vs. $R e_{\tau}$ : (red) -, $\cdots,(2.4) \pm 2 \%$; (black) - - , $11.5-19.3 R e_{\tau}^{-1 / 4}$ of CS2021; (black) - - - , 3.54 + 0.646 $\ln R e_{\tau}$ of Samie et al. (2018). Channel DNS (•): (red) DNS of table 1, (dark red) DNS of Bernardini, Pirozzoli \& Orlandi (2014), (yellow) DNS of Lozano-Durán \& Jiménez (2014). Pipe ( ): (pink) DNS of Pirozzoli et al. (2021), (blue) Superpipe NSTAP data of Hultmark et al. (2012), (purple) corrected and uncorrected $(\diamond)$ CICLoPE hot-wire data of Fiorini (2017). ZPG TBL (ם): (green) Sillero, Jiménez \& Moser (2013), (orange) Samie et al. (2018). Couette (ム): (green) Kraheberger et al. (2018).

described by

$$
\begin{aligned}
\langle u u\rangle_{\text {wall }}^{+}= & \mathcal{M}_{2}\left(y^{+} ; 0.25,373,1.7,8.8616\right)+\mathcal{H}\left(y^{+} ; 4,1.3,15\right)+ \\
& +\frac{1}{\operatorname{Re}_{\tau}^{1 / 4}}\left\{\mathcal{C}\left(y^{+} ; 3.1623,-8.4,2\right)+\mathcal{H}\left(y^{+} ;-3.7,1,13\right)\right\}+\text { HOT }
\end{aligned}
$$




\section{P.A. Monkewitz}

To go beyond $O\left(\operatorname{Re}_{\tau}^{-1 / 4}\right)$, the Reynolds number range of available DNS and their mutual consistency are insufficient.

From (2.2) and (A3), one readily obtains the Taylor expansion of $\langle u u\rangle^{+}$about the wall as

$$
\langle u u\rangle_{\text {wall }}^{+}\left(y^{+} \rightarrow 0\right)=\left(0.25-0.42 R e_{\tau}^{-1 / 4}\right)\left(y^{+}\right)^{2}-0.02\left(1-\operatorname{Re}_{\tau}^{-1 / 4}\right)\left(y^{+}\right)^{4}+\cdots .
$$

As noted above in point (i), the coefficient of $\left(y^{+}\right)^{2}$ in (2.3) fits all the $\overline{b_{1}^{2}}$ in table 1 of Hultmark \& Smits (2021) to within less than $1 \%$, which is not surprising as they used the same DNS data. The upper limit of $1 / 4$ for the coefficient of $\left(y^{+}\right)^{2}$ in $(2.3)$ is also consistent, within uncertainty, with the value of 0.26 , obtained by Monkewitz \& Nagib (2015, figure 6 and (2.19)) for the ZPG TBL.

Next, the inner peak height $\langle u u\rangle_{I P}^{+}$at $y^{+} \approx 15$ is obtained from (2.2) or figure 1 as

$$
\langle u u\rangle_{I P}^{+}=11.3-17.7 R e_{\tau}^{-1 / 4},
$$

to be compared in figure 2 with some laboratory and computational data for channel, pipe, ZPG TBL and Couette flows. Also included in the figure are the correlation $11.5-$ $19.3 R e_{\tau}^{-1 / 4}$ of CS2021, which stays within $2 \%$ of (2.4) for all $R e_{\tau}$, and $3.54+0.646 \ln R e_{\tau}$ of Samie et al. (2018), which starts to deviate more than $+2 \%$ beyond a $R e_{\tau}$ of 30000 .

While most of the experimental and DNS data are within $\pm 2 \%$ of (2.4), there are two notable exceptions: the Couette data point of Kraheberger, Hoyas \& Oberlack (2018), for which no explanation can be offered at this time, and the Superpipe NSTAP data for $R e_{\tau}>5400$. A tentative explanation for the low $\langle u u\rangle_{I P}^{+}$values in the Superpipe is given in Appendix B.

With (A2) and (A5) of Appendix A, the large $y^{+}$asymptote of the profile (2.2) is the logarithmic law

$$
\begin{gathered}
\langle u u\rangle_{\text {wall }}^{+}\left(y^{+} \gg 1\right)=S_{\text {wall }} \ln y^{+}+C_{\text {wall }} \quad \text { with } \\
S_{\text {wall }}=0.85-8.4 R e_{\tau}^{-1 / 4}, \quad C_{\text {wall }}=5.251+9.671 \operatorname{Re}_{\tau}^{-1 / 4} .
\end{gathered}
$$

The logarithmic asymptotes (2.5) of $\langle u u\rangle_{\text {wall }}^{+}$for different $R e_{\tau}$ are visualized in figure 3 by the fan of straight dashed lines intersecting at $y^{+} \approx 3$, and allow the completion of the inner expansion even though they are followed by the data only over a short interval. The logarithmic slope $S_{\text {wall }}$ of these asymptotes, negative at low $R e_{\tau}$, is seen to become positive at $R e_{\tau} \approx 10^{4}$. A short log law of $\langle u u\rangle^{+}$, albeit with a fixed slope, has already been seen by Hultmark $(2011,(4.3))$ in the same $y^{+}$-range of the Superpipe, but the role of probe corrections remains an issue (see Appendix B). More recently, Samie et al. (2018, figure 4) have developed a logarithmic fit of $\langle u u\rangle^{+}$for the region leading up to the outer peak, which, at $R e_{\tau}=20000$, is within $4 \%$ of (2.5) over the interval $10^{2} \leq y^{+} \leq 10^{3}$. This establishes the relation between the change of sign of $S_{\text {wall }}$ in (2.6) and the emergence of an outer peak in the $\langle u u\rangle^{+}$profile, known for over 20 years to appear at high $\operatorname{Re}_{\tau}$ (see, for instance, Fernholz \& Finley 1996).

To actually form such an outer peak, the wall asymptote (2.5) has to cross over to a decay law at some $y_{\times}^{+}$. This cross-over location $y_{\times}^{+}$and the decay law beyond $y_{\times}^{+}$could in principle be extracted from DNS data in a manner similar to the determination of $\langle u u\rangle_{\text {wall }}^{+}$. Due to the complexity of the expansion and the limitations of the DNS data, this has not been possible. A first indication on the value of $y_{\times}^{+}$comes from figure 1, which shows that 


\section{Streamwise Reynolds stress}

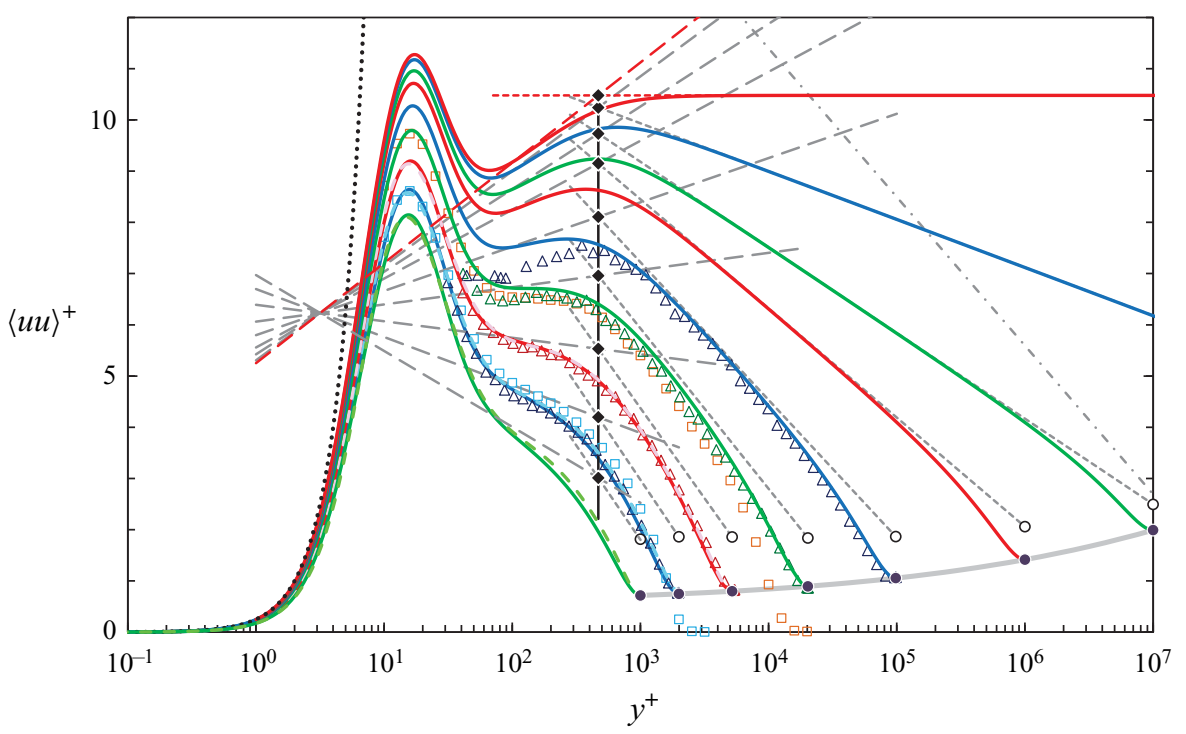

Figure 3. Solid lines: composite profiles of $\langle u u\rangle^{+}$for $R e_{\tau}=1000$ (green), 1995 (blue), 5186 (red), 20250 (green), 98190 (blue), $10^{6}$ (red), $10^{7}$ (green), $10^{9}$ (blue), $\infty$ (red). - - - DNS profiles 5 (green), 4 (blue) and 1 (red) of table 1. Symbols: $\triangle \triangle \triangle$, NSTAP Superpipe profiles of Hultmark et al. (2012) at $\operatorname{Re}_{\tau}=1985,5411$, 20250 and 98 187; $\square$, ZPG TBL profile of Sillero et al. (2013) for $R_{\tau}=1989$ (blue) and of Samie et al. (2018) for $R e_{\tau}=14500$ (orange). Note that TBL profiles have not been used for the construction of the composite expansion and are shown for comparison only. Long grey dashes (red for $R e_{\tau}=\infty$ ), wall $\log \operatorname{laws}(2.5)$ for the $11 R e_{\tau}$; short grey dashes (red for $R e_{\tau}=\infty$ ), corresponding overlap log laws (2.9) with $\circ$ marking their 'end points' at $y^{+}=R e_{\tau} ; \diamond$, intersections of wall and overlap log laws at $y_{\times}^{+}(2.7)$; - (grey), fit (3.3) of $\langle u u\rangle_{C L}^{+}$, with - marking the fit at the $R e_{\tau}$ values of the profiles shown; $\cdots$, leading term $0.25\left(y^{+}\right)^{2}$ of the Taylor expansion about the wall; $-\cdot-$, logarithmic slope of -1.26 .

the channel DNS closely follows the wall $\log$ law $(2.5)$ up to $y^{+} \approx 200$, implying that $y_{\times}^{+}$ must be larger than 200 .

Turning to a straight fit of $y_{\times}^{+}$, all the data between $R e_{\tau}=10^{3}$ and $10^{5}$ are seen in figure 3 to be compatible with $y_{\times}^{+}$equal to a simple constant

$$
y_{\times}^{+}=470 \quad \text { and } \quad\langle u u\rangle_{\times}^{+} \equiv\langle u u\rangle_{\text {wall }}^{+}\left(y_{\times}^{+}\right)=10.48-42.0 R e_{\tau}^{-1 / 4},
$$

with the corresponding $\langle u u\rangle_{\times}^{+}$following from ((2.5), (2.6)). To guide the eye, the points $\left(y_{\times}^{+},\langle u u\rangle_{\times}^{+}\right)$are marked by $\checkmark$ for the profiles of figure 3 . It is important to note here, that $\langle u u\rangle_{\times}^{+}$is not the outer maximum nor $y_{\times}^{+}$its location, but the intersection of the logarithmic asymptote (2.5) with the asymptotic logarithmic decay law of the overlap region. As the actual outer peak height and its location depend on the slopes of both asymptotes, and on the manner the corner between them is smoothed, its detailed discussion is postponed to the short $\S 4$, after the complete composite expansion is established in $\S 3$.

The adoption of a constant $y_{\times}^{+}$for all $R e_{\tau}$ means that the inner expansion, which cannot end at a finite value of the inner coordinate, extends beyond the cross-over point $y_{\times}^{+}$into the region of logarithmic decay, where it overlaps with the outer expansion. Hence, the complete inner expansion is obtained by adding a corner function (A5) to $\langle u u\rangle_{\text {wall }}^{+}$of (2.2)

$$
\langle u u\rangle_{\text {in }}^{+}=\langle u u\rangle_{\text {wall }}^{+}+\mathcal{C}\left(y^{+} ; y_{\times}^{+}, \Delta S, 2\right)
$$




\section{P.A. Monkewitz}

and its limit for $y^{+} \gg y_{\times}^{+} \gg 1$ yields the asymptotic logarithmic overlap layer, i.e. the common part of inner and outer expansions

$$
\begin{aligned}
\langle u u\rangle_{c p}^{+} \equiv\langle u u\rangle_{\text {in }}^{+}\left(y^{+} \gg y_{\times}^{+} \gg 1\right) & =S_{c p}\left[\ln y^{+}-\ln y_{\times}^{+}\right]+\langle u u\rangle_{\times}^{+}+\text {HOT } \\
\text { with } \quad S_{c p} & =S_{\text {wall }}+\Delta S .
\end{aligned}
$$

The logarithmic slope $S_{c p}$ of the overlap log law (2.9) must be determined by matching to the outer expansion in $\S 3$. At this point it can only be said that $S_{c p}$ must be negative to form an outer peak at large $R e_{\tau}$. Furthermore, it must go to zero at infinite $R e_{\tau}$, as shown in figure 3, because $\langle u u\rangle^{+}$near the wall has been shown to remain finite for all $R e_{\tau}$. This implies that $\Delta S$ is of the form $\Delta S=-0.85+\Delta S^{\prime}$, with $\Delta S^{\prime}$ vanishing for $R e_{\tau} \rightarrow \infty$, to compensate the $O(1)$ contribution to $S_{\text {wall }}$ in (2.6).

\section{The outer and composite expansions of $\langle u u\rangle^{+}$}

Moving on to the outer expansion, it is written as a logarithmic part matching the common part (2.9) for small $Y$ and satisfying the symmetry condition on the centreline $Y=1$, plus a wake part $\mathcal{W}(Y)$, which goes to zero for $Y \rightarrow 0$

$$
\langle u u\rangle_{\text {out }}^{+}=S_{\text {out }} \ln \left[\operatorname{Re}_{\tau} Y\left(1-\frac{Y}{2}\right)\right]-S_{\text {out }} \ln y_{\times}^{+}+\langle u u\rangle_{\times}^{+}+\mathcal{W}(Y)+\text { HOT. }
$$

The matching of outer and inner expansions furthermore requires $S_{\text {out }}$ to be identical to $S_{c p}$ in (2.9).

To obtain the logarithmic slope $S_{\text {out }}=S_{c p}$, the outer expansion (3.1) is evaluated at $Y=1$ and identified with the fit (3.3) of centreline stress

$$
\begin{aligned}
\langle u u\rangle_{C L}^{+} & =S_{\text {out }} \ln \left(\frac{R e_{\tau}}{2}\right)-S_{\text {out }} \ln y_{\times}^{+}+\langle u u\rangle_{\times}^{+}+\mathcal{W}(1) \\
& =0.55+\left[0.1007+33 R e_{\tau}^{-1 / 4}\right]^{-1} .
\end{aligned}
$$

As seen in figure 3, this fit reproduces the channel and Superpipe centreline data up to $R e_{\tau}=10^{5}$. At higher $R e_{\tau},\langle u u\rangle_{C L}^{+}$increases to the infinite Reynolds number limit of $\langle u u\rangle_{\times}^{+}=10.48$, such that $\langle u u\rangle_{\text {out }}^{+}$becomes a simple constant throughout the channel or pipe. Note that the fit (3.3), which relies strongly on the outer Superpipe data of Hultmark et al. (2012), implies that differences are relatively minor between the outer expansions for pipe and channel (see comments in $\S 5$ ).

What is still missing for the determination of the outer logarithmic slope $S_{\text {out }}$ is the wake function $\mathcal{W}(Y)$. The fit with the requisite symmetry properties

$$
\mathcal{W}(Y)=S_{\text {out }} \ln \left[\frac{1}{4}+\frac{3}{4}(1-Y)^{2}\right],
$$

is again developed from both channel DNS and Superpipe NSTAP data, while ZPG TBLs obviously require a different $\mathcal{W}(Y)$. However, it appears from figure 3, that the TBL overlap log law remains close to (2.9) developed for channel and pipe.

Equation (3.4), together with (3.2) and (3.3), finally allows one to determine $S_{\text {out }}=S_{c p}$ in (2.9). The resulting logarithmic slope is found to scale as

$$
S_{\text {out }}=\sigma \ln ^{2} \operatorname{Re}_{\tau} \operatorname{Re}_{\tau}^{-1 / 4}
$$

with $\sigma$ a weak function of $R e_{\tau}$, varying between -0.19 and -0.15 in the interval $R e_{\tau} \in\left[10^{3}, 10^{10}\right]$. While the $\operatorname{Re}_{\tau}^{-1 / 4}$ dependence is directly related to the scaling of $\langle u u\rangle_{i n}^{+}$, 


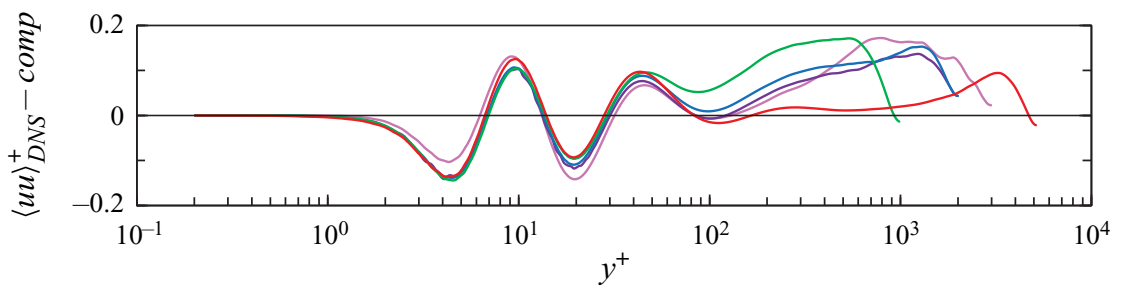

Figure 4. Difference between the DNS profiles $\langle u u\rangle^{+}$of table 1 ( 1-5: red, pink, violet, blue, green) and their complete composite fit (3.6).

obtained without model assumptions, the factor $\ln ^{2} R e_{\tau}$ in (3.5) may depend on the details of how $S_{\text {out }}$ has been determined. However, as long as $\langle u u\rangle_{\times}^{+}$remains finite, $S_{\text {out }}$ must go to zero for $R e_{\tau} \rightarrow \infty$.

With the determination of $S_{\text {out }}$, the composite expansion

$$
\langle u u\rangle_{\text {comp }}^{+}=\langle u u\rangle_{\text {in }}^{+}+\langle u u\rangle_{\text {out }}^{+}-\langle u u\rangle_{c p}^{+},
$$

is complete up to $O\left(\operatorname{Re}_{\tau}^{-1 / 4}\right)$. This final result is compared in figure 4 with the DNS data of table 1 , and the differences between composite expansion and DNS are seen to be at most $1.5 \%$ of the inner peak height (2.4). It is also noted that, in the region $0 \leq y^{+} \lesssim 10^{2}$, the difference between DNS and composite expansion is principally due to an imperfect 'hump' function (A4). However, no improvement is pursued here, as the deviations from DNS are barely larger than the line thickness in figure 1 and no additional insight would be gained from a more complex $\mathcal{H}$.

The composite expansion (3.6) allows a unified comparison with complete $\langle u u\rangle^{+}$ profiles of different origins, as well as an extrapolation of $\langle u u\rangle^{+}$to truly large $\operatorname{Re}_{\tau}$. Figure 3 shows the close correspondence, over the entire $R e_{\tau}$ range of $10^{3}$ to $10^{5}$, between composite profiles and both DNS and several more recent high Reynolds number laboratory data. Only the Superpipe data of Hultmark et al. (2012) are seen to progressively fall below the composite expansion close to the wall, which is also reflected in the low inner peak heights for the Superpipe in figure 2. An explanation for these discrepancies is proposed in Appendix B.

Figure 3 also shows the asymptotic 'skeleton' of the composite expansion: the fan of logarithmic asymptotes (2.5) of $\langle u u\rangle_{\text {wall }}^{+}(2.2)$ and the corresponding asymptotic overlap log laws (2.9), together with their intersections (2.7), marked by $\boldsymbol{\nabla}$. The considerable difference, at the lower Reynolds numbers, between this asymptotic logarithmic 'skeleton' and the composite expansion, is already noted here. Similarly, the data are seen to closely approach the overlap log law (2.9) - the short-dashed grey lines between $\checkmark$ and $\circ$ in figure 3 - only beyond a $R e_{\tau}$ of approximately $10^{5}$. Below this $R e_{\tau}$, the wake region is reached before the condition $y^{+} \gg y_{\times}^{+}$is satisfied. See also the comments in the concluding $\S 5$ and the comparison with the patched asymptotic expansion of Marusic \& Kunkel (2003) in figure 3 of the supplementary material.

\section{The outer peak}

The scaling of the outer peak $\langle u u\rangle_{O P}^{+}$and its location $y_{O P}^{+}$have given and still give rise to extended debates, because they are not yet accessible to DNS and in experiments are typically seen at a wall distance where probe corrections are often an important issue. Probably the first, clean characterization of this peak has been provided by Samie et al. (2018) for the ZPG TBL at $R e_{\tau}$ values up to 20'000. The Reynolds number 


\section{P.A. Monkewitz}
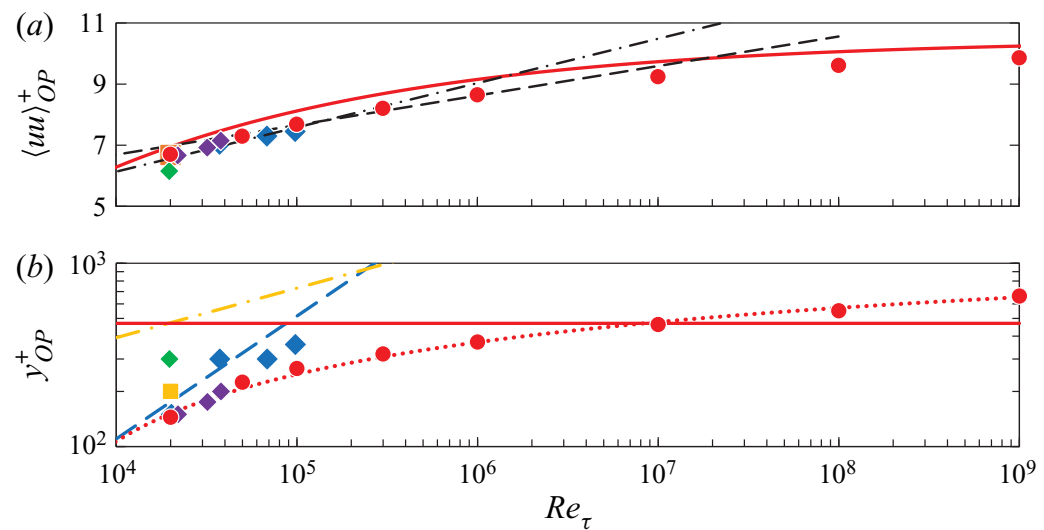

Figure 5. (a) Outer peak height $\langle u u\rangle_{O P}^{+}$vs $R e_{\tau}$. Symbols as in figure 2, except for: green $\diamond$, pipe data point of Morrison et al. (2004); red •, outer peak height of full composite expansion (3.6) at selected $\operatorname{Re}_{\tau}$ values; red - $\langle u u\rangle_{\times}^{+}$of (2.7); black - - - and - - - - , outer peak correlations $2.82+0.42 \ln R e_{\tau}$ and $0.33+$ $0.63 \ln R e_{\tau}$ of Pullin, Inoue \& Saito (2013). (b) Corresponding outer peak locations $y_{O P}^{+}$. Symbols as in panel $(a)$, except for: red —, $y_{\times}^{+}=470$; blue - - , correlation of Hultmark et al. (2012); red - . , fit $1200-$ $1900 R e_{\tau}^{-0.06}$; yellow $-\cdot-\cdot-$, correlation $32.66 R e_{\tau}^{0.27}$ of Samie et al. $(2018,(3.3 b))$ for the intersection of tangents outside of $y_{O P}^{+}$.

dependence of the outer peak, observed by Samie et al. (2018), appears to clash with the choice of a constant $y_{\times}^{+}$in (2.7). A close look at figure 3 shows, however, that the location $y_{O P}^{+}$of the actual outer peak depends strongly on the slopes of the wall and overlap log laws (2.5) and (2.9), as well as on how the transition between the two is fitted. This sensitivity to fitting details is comparable to the sensitivity to measurement errors in experiments (see also Appendix B).

The evolutions of the outer peak height $\langle u u\rangle_{O P}^{+}$and location $y_{O P}^{+}$with $R e_{\tau}$, obtained from the present composite expansion (3.6), are shown in figure 5; $\langle u u\rangle_{O P}^{+}$, which is always below $\langle u u\rangle_{\times}^{+}(2.7)$, is seen in panel (a) to be fully consistent with other experimental data, as well as with the outer peak correlations $2.82+0.42 \ln R e_{\tau}$ and $0.33+0.63 \ln R e_{\tau}$ of Pullin et al. (2013), up to $R e_{\tau}$ values well above $10^{6}$.

Panel (b) shows the location $y_{O P}^{+}$vs $R e_{\tau}$. In this panel, the uncertainty of the experimental points is large and could be as high as $100 \%$ at the lower $\operatorname{Re}_{\tau}$ values. Up to $R e_{\tau}=10^{5}$ the outer peak locations for both the data and the present composite expansion are seen to be compatible with the correlation $\propto R e_{\tau}^{0.67}$ of Hultmark et al. (2012). Samie et al. $(2018,(3.3 b))$, on the other hand, give a correlation of $32.66 R e_{\tau}^{0.27}$ for the intersection of two logarithmic tangents to the data, which is located outside of the outer peak, and provides an upper bound on $y_{O P}^{+}$. Together with the outer peak location of the present composite expansion, which is well fitted by the ad hoc correlation $y_{O P}^{+}=1200-1900 R e_{\tau}^{-0.06}$, the outer peak is firmly placed in the inner asymptotic region, meaning that, in terms of the usual intermediate or overlap variable $\eta=y^{+} / \operatorname{Re}_{\tau}^{1 / 2}$, the outer peak approaches $\eta=0$ in the limit of $R e_{\tau} \rightarrow \infty$.

\section{Discussion and outlook}

In conclusion, the first complete composite profile of $\langle u u\rangle^{+}$, based on the inner asymptotic sequence $\left\{1, \operatorname{Re}_{\tau}^{-1 / 4}, \ldots\right\}$ proposed by CS2021, provides a rather satisfactory description 


\section{Streamwise Reynolds stress}

of DNS and experimental $\langle u u\rangle^{+}$profiles for Reynolds numbers between $10^{3}$ and $10^{5}$, as seen in figure 3. What is curious, however, is that CS2021 and Smits et al. (2021) fully agree on the proportionality between the inner peak height $\langle u u\rangle_{I P}^{+}$and the coefficient of $\left(y^{+}\right)^{2}$ in the Taylor expansion of $\langle u u\rangle^{+}$about the wall and even on the value of their ratio, while completely disagreeing on the Reynolds number scaling of the two quantities. Furthermore, the $\operatorname{Re}_{\tau}^{-1 / 4}$ scale, or any other scale, apart from $\operatorname{Re}_{\tau}^{-1}$, does not appear naturally in the Reynolds stress transport equation for $\langle u u\rangle^{+}$. What is particularly intriguing is that the exact production term for channel flow, $\mathcal{P}^{+}=\left(d U^{+} / d y^{+}\right)(1-$ $\left.d U^{+} / d y^{+}-y^{+} / R e_{\tau}\right)$, does not vary as $\operatorname{Re}_{\tau}^{-1 / 4}$, but has an inner asymptotic expansion of the form $\mathcal{P}_{0}^{+}\left(y^{+}\right)+R e_{\tau}^{-1} \mathcal{P}_{1}^{+}\left(y^{+}\right)+\cdots$ (see Monkewitz 2021).

If the correct expansion parameter was indeed $\left(1 / R e_{\tau}\right)$, clearly more than two terms would be required in the inner expansion of $\langle u u\rangle^{+}$for a good approximation at the Reynolds numbers of the DNS data in table 1 , that is

$$
\langle u u\rangle^{+} \approx f\left(y^{+}\right)+g\left(y^{+}\right) R e_{\tau}^{-1 / 4} \approx f\left(y^{+}\right)+\sum_{n=1}^{N} h_{n}\left(y^{+}\right) R e_{\tau}^{-n} .
$$

This is illustrated for the inner peak height in figure $6(a)$, which is figure 2 replotted against $R e_{\tau}^{-1}$, with the addition of the relatively simple fit

$$
\langle u u\rangle_{I P}^{+}=7.6+1.9 \exp \left(\frac{-1300}{\operatorname{Re}_{\tau}}\right)+\exp \left(\frac{-15000}{\operatorname{Re}_{\tau}}\right)=10.5-\frac{1.7510^{4}}{\operatorname{Re}_{\tau}}+O\left(\operatorname{Re}_{\tau}^{-2}\right),
$$

and its two-term Taylor expansion. This fit is seen to describe the Reynolds number dependence of all the $\langle u u\rangle_{I P}^{+}$data included in figure 2 as well as the other correlations, and its two-term Taylor expansion provides an excellent fit for $R e_{\tau} \gtrsim 2.10^{4}$. Figure $6(b)$ shows that the fit (5.2) divided by 46 is again an excellent match for the coefficient of $\left(y^{+}\right)^{2}$ in the Taylor expansion about the wall. However, one immediately notices that this coefficient only reaches 0.228 instead of the 0.25 inferred by CS2021. This discrepancy may well be due to the simplifying assumptions of CS2021. Actually, the ratio between the production at $y_{I P}^{+} \approx 15$ and the turbulent transport at the wall in the DNS 1 of table 1 is 1.12 , tantalizingly close to the ratio $0.25 / 0.228=1.10$. Finally, the fact that the first two terms of the Taylor expansion in (5.2) require a $R e_{\tau}$ in excess of $10^{4}$ to provide a good approximation of the full exponential fit is reminiscent of the behaviour of the indicator function $\Xi^{+}=y^{+}\left(\mathrm{d} U^{+} / \mathrm{d} y^{+}\right)$in figure 12 of Monkewitz (2021), which starts to reach the correct log-law plateau only for Reynolds numbers around $10^{5}$.

Beyond these speculations on scaling, which require more thought, the features of the $\langle u u\rangle^{+}$profiles, shown in figure 3, call for the following comments and conclusions:

(i) The analysis of the channel DNS profiles of table 1 in $\S 2$ has demonstrated, that the structure of the inner asymptotic expansion of $\langle u u\rangle^{+}$does not change between the wall and $y^{+} \approx 200$, and supports the somewhat complex argument of CS2021 that $\langle u u\rangle^{+}$remains finite in the limit of infinite Reynolds number. It has furthermore demonstrated that the coefficient of $\left(y^{+}\right)^{2}$ in the Taylor expansion of $\langle u u\rangle^{+}$about the wall and the inner peak height $\langle u u\rangle_{I P}^{+}$at $y^{+} \approx 15$ are proportional, or nearly so, which has been fully confirmed by the data analyses of Hultmark \& Smits (2021) and Smits et al. (2021).

(ii) The detailed analysis of the transport equation for $\langle u u\rangle^{+}$in $\S 2$ has shown that the unlimited growth of $\langle u u\rangle_{I P}^{+}$with $\ln R e_{\tau}$, predicted by the attached eddy model 


\section{P.A. Monkewitz}

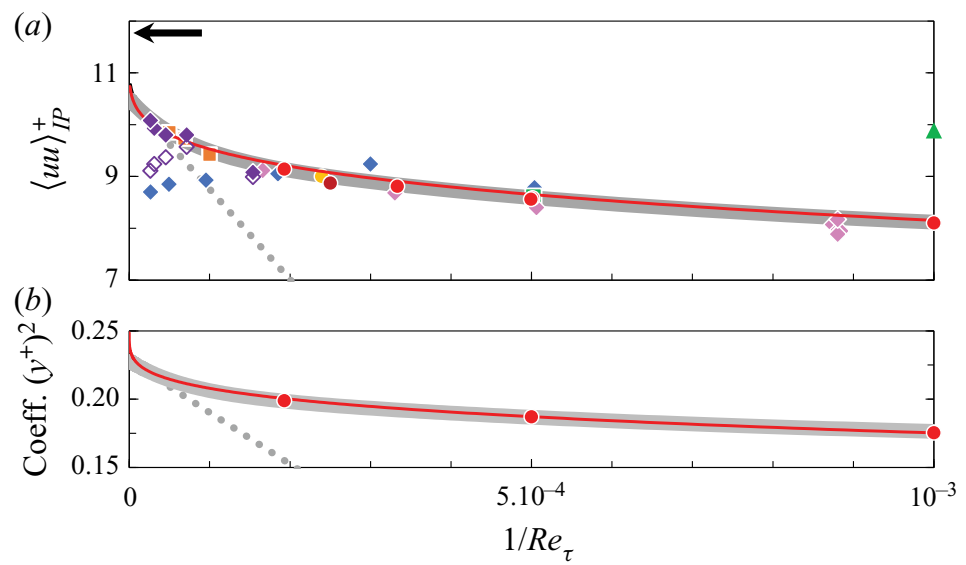

Figure 6. (a) Inner peak height $\langle u u\rangle_{I P}^{+}$vs $\left(1 / R e_{\tau}\right)$ with same data as in figure 2. Lines: (red) -, (2.4); (wide grey) -, (5.2); (grey) $\cdots$, two-term Taylor expansion of 'grey' fit; $\leftarrow$, lower end of the range of the atmospheric inner peak data of Metzger \& Klewicki (2001) at $R e_{\tau} \approx 8.10^{5}$. (b) Coefficient of $\left(y^{+}\right)^{2}$ in the Taylor expansion of $\langle u u\rangle^{+}$about the wall: (red) $\bullet$, DNS data 1, 4 and 5 of table 1; (red) —, (2.3); (wide grey) —, (5.2) divided by 46 ; (grey) $\cdots$, two-term Taylor expansion $0.228-380 / R e_{\tau}$ of 'grey' fit.

(Marusic \& Monty 2019) is very unlikely. The possibility of an unlimited growth of the outer peak with $R e_{\tau}$ appears equally unlikely, considering that the cross-over location $y_{\times}^{+}$from the wall log law (2.5) to the overlap log law not only scales on inner units, but remains constant over the $R e_{\tau}$ range where laboratory data are available, as seen in figure 3 .

(iii) As discussed in $\S 4$, the present composite profiles in figure 3 also match, within the considerable uncertainty, the height and location of distinct outer peaks reported in the literature, with the notable exception of some NSTAP data discussed in Appendix B.

(iv) With both inner and outer peaks of $\langle u u\rangle^{+}$finite, a simple geometric argument, already brought up by Monkewitz \& Nagib (2015), rules out the Reynolds-independent slope of the overlap log law in the attached eddy model (see figure 3 in $\S 2$ of the supplementary material). Due to its relatively simple structure, it appears nevertheless useful, if parameters are adapted to the Reynolds number range under consideration.

(v) The switch over from the wall log law to the asymptotic overlap log law at a fixed value of $y_{\times}^{+} \approx 470$ is reminiscent of the change of logarithmic slope from $1 / 0.398$ to $1 / 0.42$ at $y^{+}=624$, found by Monkewitz (2021) in the mean velocity profile and tentatively interpreted as an opposite wall effect. A connection between the two observations is conceivable, but the mechanism remains to be elucidated.

(vi) Also open is the question whether the $\langle u u\rangle^{+}$composite expansion developed here is universal or not, excluding of course the wake region in ZPG TBLs. For the near-wall region in figure 1, the present expansion relies entirely on channel DNS, while at the higher $R e_{\tau}$, the outer part of the Superpipe profiles of Hultmark et al. (2012) has helped guide the expansion. The close correspondence in figure 3 between the channel DNS and Superpipe profiles for $R e_{\tau}$ of 1985 and 5411 suggests that the differences between channel and pipe are small. It would, however, be surprising if there were no differences at all, at least in the outer $\langle u u\rangle^{+}$expansion, as there are strong indications (Monkewitz 2021) that the outer mean velocity expansions, in 


\section{Streamwise Reynolds stress}

particular the Kármán 'constants', are different for channel and pipe. In ZPG TBLs, finally, $\langle u u\rangle^{+}$also appears to remain close to channel and pipe, up to and including the overlap layer. However, the present asymptotic expansion underestimates the inner peak heights in the atmospheric data of Metzger \& Klewicki (2001), indicated in figure 6(a) by an arrow, but no attempt has been made here to untangle the possible reasons.

(vii) Finally, it must be reiterated that determining the slopes of log laws, which are inherently asymptotic laws, by fitting tangents to finite Reynolds number data is hazardous. As illustrated in figure 3, only at the highest NSTAP $R e_{\tau}$ of around $10^{5}$ does the overlap log law start to go through the data! This is the same conclusion as the one reached by Monkewitz (2021, figure 12) and Spalart \& Abe (2021, see their extrapolation in figure $3 b$ ), who found that the mean velocity indicator function $y^{+}\left(\mathrm{d} U^{+} / \mathrm{d} y^{+}\right)$starts to reach the correct log-law plateau only beyond a $R e_{\tau}$ of around $10^{5}$. Up to such high $R e_{\tau}$, the development of proper asymptotic expansions is indispensable.

Supplementary material. Supplementary material are available at https://doi.org/10.1017/jfm.2021.924.

Acknowledgements. I am grateful to K. 'Sreeni' Sreenivasan, H. Nagib, X. Chen and an anonymous reviewer for their helpful comments and encouragement. Thanks also to M. Hultmark for the stimulating discussion on Appendix B.

Declaration of interest. The author reports no conflict of interest.

Author ORCIDs.

(1) Peter A. Monkewitz https://orcid.org/0000-0003-4279-725X.

\section{Appendix A. The fit for the near-wall $\langle u u\rangle^{+}$profile and other fits}

Inspired by the methodology of Musker (1979) for the mean velocity fit, the $O(1)$ part of the inner (near-wall) $\langle u u\rangle^{+}$Reynolds stress profile is approximated analytically by the integral of $\mathrm{d} \mathcal{M}_{2} / \mathrm{d} y^{+}=2 p_{0} y^{+}\left[p_{1}+p_{2}\left(y^{+}\right)^{2}\right]\left[p_{1}+p_{3}\left(y^{+}\right)^{2}+\left(y^{+}\right)^{4}\right]^{-1}$, where the subscript ' 2 ' indicates that the leading term of the Taylor expansion of $\mathcal{M}_{2}$ around $y^{+}=0$ is $\propto\left(y^{+}\right)^{2}$. The result of the integration is

$$
\begin{aligned}
\mathcal{M}_{2}\left(y^{+} ; p_{0}, p_{1}, p_{2}, p_{3}\right)= & \frac{p_{0}\left(2 p_{1}-p_{2} p_{3}\right)}{\sqrt{4 p_{1}-p_{3}^{2}}}\left\{\arctan \frac{p_{3}+2\left(y^{+}\right)^{2}}{\sqrt{4 p_{1}-p_{3}^{2}}}-\arctan \frac{p_{3}}{\sqrt{4 p_{1}-p_{3}^{2}}}\right\} \\
& +\frac{p_{0} p_{2}}{2} \ln \left[1+\frac{p_{3}\left(y^{+}\right)^{2}}{p_{1}}+\frac{\left(y^{+}\right)^{4}}{p_{1}}\right],
\end{aligned}
$$

with the parameters $p_{0} \cdots p_{3}$ determined by the boundary conditions. For large $y^{+}, \mathcal{M}_{2}$ asymptotes to the log law

$$
\mathcal{M}_{2}\left(y^{+} \gg 1\right)=\frac{p_{0} p_{2}}{2}\left\{4 \ln \left(y^{+}\right)-\ln \left(p_{1}\right)\right\}+\frac{p_{0}}{\sqrt{4 p_{1}-p_{3}^{2}}}\left\{\frac{\pi}{2}-\arctan \frac{p_{3}}{\sqrt{4 p_{1}-p_{3}^{2}}}\right\},
$$

and near the wall it has the Taylor expansion

$$
\mathcal{M}_{2}\left(y^{+} \rightarrow 0\right)=p_{0}\left(y^{+}\right)^{2}+\frac{p_{0}\left(p_{2}-2 p_{3}\right)}{2 p_{1}}\left(y^{+}\right)^{4}+\cdots .
$$




\section{P.A. Monkewitz}

Like the original mean velocity Musker profile, the profile (A1) misses a 'hump' centred around $y^{+}=O(10)$. As in Monkewitz (2021), it is modelled by the 'hump' function of Nagib \& Chauhan (2008)

$$
\mathcal{H}\left(y^{+} ; h_{1}, h_{2}, h_{3}\right)=h_{1} \exp \left[-h_{2} \ln ^{2}\left(y^{+} / h_{3}\right)\right] .
$$

Finally, the smooth transition between two logarithmic laws with different slopes in a variable $\eta$ is fitted by the corner function

$$
\mathcal{C}\left(\eta ; \eta_{c}, c, m\right)=\frac{c}{m} \ln \left[1+\left(\frac{\eta}{\eta_{c}}\right)^{m}\right] \rightarrow \begin{cases}0 & \text { for } \eta \ll \eta_{c} \\ c\left[\ln \eta-\ln \eta_{c}\right] & \text { for } \eta \gg \eta_{c}\end{cases}
$$

with the rounding of the corner at $\eta_{c}$ governed by the parameter $m$.

\section{Appendix B. Survey of the effect of wall proximity on NSTAP measurements}

The debate on whether near-wall statistics in turbulent wall-bounded flows are universal or not depends crucially on experimental data at high Reynolds numbers. Focussing on the streamwise Reynolds stress, the problem is exemplified by the discrepancies between measured inner peak heights $\langle u u\rangle_{I P}^{+}$in figure 2. Of particular interest is the marked difference between the CICLoPE data of Fiorini (2017) and the Superpipe data of Hultmark et al. (2012), both corrected for spatial probe averaging. Since the corrections (shown in the figure for the CICLoPE data) are rather large and the probes very different (standard hot-wire vs NSTAP, described in Vallikivi \& Smits 2014), it has not yet been possible to explain the origin of the discrepancy.

To advance the discussion, the NSTAP measurements in two different ZPG TBL facilities are compared, one in the Melbourne wind tunnel at atmospheric pressure by Samie et al. (2018) and the other in the pressurized Princeton facility by Vallikivi et al. (2015) (available from https://smits.princeton.edu). The comparison is shown in figure 7 and reveals a significant difference between the two $\langle u u\rangle^{+}$data sets below a $y^{+}$of the order of $10^{2}$. Looking for corresponding differences of operating parameters in the two set-ups, the physical wall distance of the sensing element is the most conspicuous. In the pressurized Princeton facility, a wall distance of $300 \mu \mathrm{m}$ corresponds to $y^{+}$of 90 and more, indicated in figure 7 by vertical dashed lines for the three $R e_{\tau}$ values. In the Melbourne tunnel, the same $300 \mu \mathrm{m}$ correspond to $y^{+} \leq 17$ for the similar $R e_{\tau}$ values of Samie et al. (2018). Lacking any other credible candidates, an explanation based on the difference between length scales $\hat{v} / \hat{u}_{\tau}$ is proposed.

The fact that the $300 \mu \mathrm{m}$ wall distance, below which the discrepancies emerge, is much larger than the NSTAP sensing element, which is a 'flat plate' of roughly $0.1 \mu \mathrm{m}$ thickness and $1 \mu \mathrm{m}$ chord, suggests that the 'culprit' may be the NSTAP silicon support structure, with overall dimensions of $4 \times 2.1 \mathrm{~mm}$ and a thickness increasing downstream to $0.5 \mathrm{~mm}$. The difference between the $\langle u u\rangle^{+}$of Samie et al. (2018) and those of Vallikivi et al. (2015) in figure 7 is seen to reach about one for $y^{+}$in the range of 20-30. In the same region, the mean velocities of Vallikivi et al. (2015) are clearly above the DNS profile of Sillero et al. (2013), again with a difference of order unity, while those of Samie et al. (2018), not shown in the figure for clarity, are slightly below the DNS. This behaviour suggests a blockage effect of the NSTAP probe body when the volume between the two wedge shaped silicon sensor supports is progressively closed off by the wall, leading at the sensor location to a mean flow acceleration at the expense of turbulence intensity.

For comparison, the deviation of the Superpipe NSTAP data of Hultmark et al. (2012) from the composite profile (3.6) is shown in figure 8 and the similarity with the deviations 


\section{Streamwise Reynolds stress}

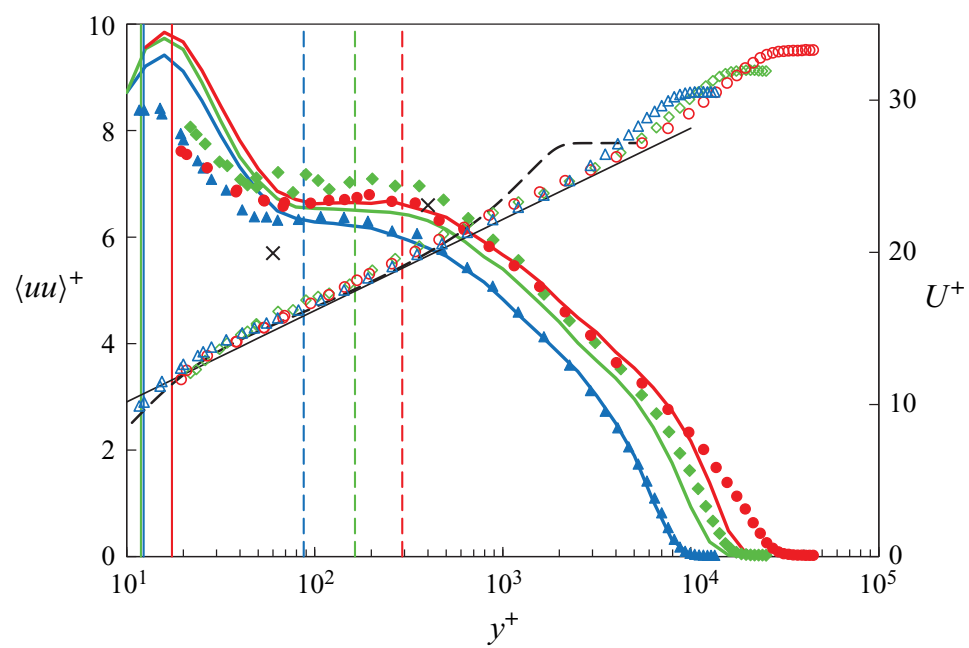

Figure 7. Comparison of NSTAP profiles of $\langle u u\rangle^{+}$and $U^{+}$in different ZPG TBL facilities. Solid lines: $\langle u u\rangle^{+}$ of Samie et al. (2018) at $R e_{\tau}=10000$ (blue), 14500 (green) and 20000 (red); vertical solid lines: $y^{+}$locations corresponding to a wall distance of $300 \mu \mathrm{m}$. Symbols: $\langle u u\rangle^{+}$of Vallikivi, Hultmark \& Smits (2015) at $R e_{\tau}=$ 8260 (blue $\boldsymbol{\Delta}$ ), 14700 (green $\$$ ) and 25000 (red •) and corresponding $U^{+}$profiles (open symbols); vertical dashed lines: $y^{+}$locations corresponding to wall distance of $300 \mu \mathrm{m} . \times$ : local minimum and outer maximum of $\langle u u\rangle^{+}$from figure 43 of Fernholz \& Finley (1996) for $R e_{\tau} \approx 18000\left(\operatorname{Re}_{\delta 2}=57720\right) ;---, U^{+}$profile of Sillero et al. (2013) for $R e_{\tau}=1989 ;-,(1 / 0.384) \ln R e_{\tau}+4.17$.

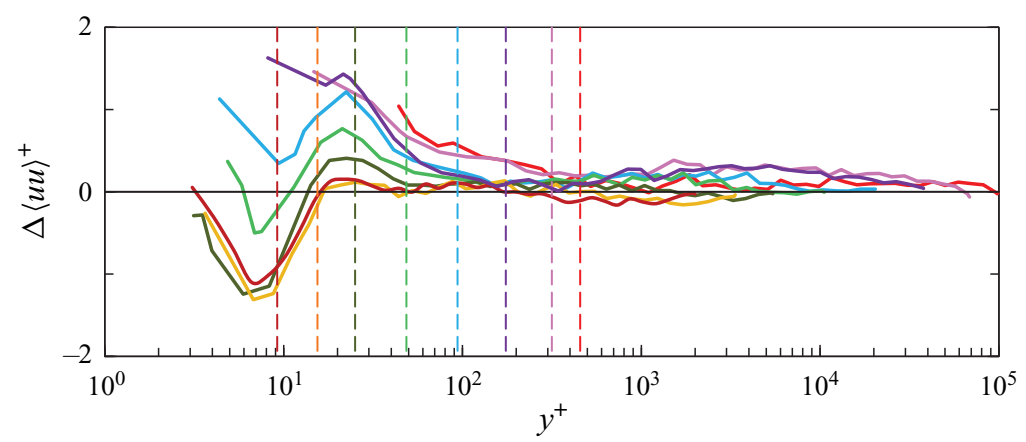

Figure 8. Difference between the composite expansion (3.6) and eight Superpipe NSTAP profiles of $\langle u u\rangle^{+}$ (Hultmark et al. 2012), from $R e_{\tau}=98187$ to 1985 (available from https://smits.princeton.edu); colour sequence red, pink, violet, light blue, light green, dark green, orange, brown. Vertical - - -, $y^{+}$values corresponding to $300 \mu \mathrm{m}$ wall distance for each $R e_{\tau}$.

between the Princeton and Melbourne ZPG TBL data, shown in figure 7, is obvious. This supports the idea that the $\langle u u\rangle^{+}$profiles in pipes, ZPG TBLs and most probably channels are very similar, possibly even identical, well into the overlap region beyond $y_{\times}^{+}$ $(\checkmark$ in figure 3 ). However, it should be noted that the NSTAP pipe data in figure 8 are being compared with a composite expansion developed mainly on lower Reynolds number channel DNS and that the suspected origin of the discrepancy - near-wall blockage by the NSTAP silicon probe support, likely also dependent on its angle of attack - will have to be tested experimentally. 


\section{P.A. Monkewitz}

\section{REFERENCES}

Bernardini, M., Pirozzoli, S. \& Orlandi, P. 2014 Velocity statistics in turbulent channel flow up to $R e_{\tau}=4000$. J. Fluid Mech. 742, 171-191.

Chauhan, K.A., Monkewitz, P.A. \& NagiB, H.M. 2009 Criteria for assessing experiments in zero pressure gradient boundary layers. Fluid Dyn. Res. 41 (2), 021404.

CHEN, X. \& SREEnivasan, K.R. 2021 Reynolds number scaling of the peak turbulence intensity in wall flows. J. Fluid Mech. 908, R3.

DEGRAAFF, D.B. \& EATON, J.K. 2000 Reynolds-number scaling of the flat-plate turbulent boundary layer. J. Fluid Mech. 422, 319-346.

FERnholZ, H.H. \& Finley, P.J. 1996 The incompressible zero-pressure-gradient turbulent boundary layer: an assessment of the data. Prog. Aerosp. Sci. 32, 245-311.

FIORINI, T. 2017 Turbulent pipe flow - high resolution measurements in CICLoPE. PhD thesis, University of Bologna.

HinzE, J.O. 1975 Turbulence, 2nd edn. McGraw-Hill.

Hoyas, S. \& JiméneZ, J. 2006 Scaling of the velocity fluctuations in turbulent channels up to $R e_{\tau}=2003$. Phys. Fluids 18, 011702.

Hultmark, M. 2011 Reynolds number effects on turbulent pipe flow. PhD thesis, Princeton University.

Hultmark, M. \& Smits, A.J. 2021 Scaling turbulence in the near-wall region. arXiv:2103.01765v1.

Hultmark, M., Vallikivi, M., Bailey, S.C. \& Smits, A.J. 2012 Turbulent pipe flow at extreme Reynolds numbers. Phys. Rev. Lett. 108, 094501.

Kevorkian, J. \& Cole, J.D. 1981 Perturbation Methods in Applied Mathematics. Springer.

Kraheberger, S., HoyAs, S. \& OBERlack, M. 2018 DNS of a turbulent Couette flow at constant wall transpiration up to $R e_{\tau}=1000$. J. Fluid Mech. 835, 421-443.

LEE, M. \& Moser, R.D. 2015 Direct numerical simulation of turbulent channel flow up to $R e_{\tau}=5200$. J. Fluid Mech. 774, 395-415.

LOZANO-DuRÁn, A. \& JimÉnEZ, J. 2014 Effect of the computational domain on direct numerical simulations of turbulent channels up to $R e_{\tau}=4200$. Phys. Fluids 26, 011702.

Marusic, I. \& KUnKel, G.J. 2003 Streamwise turbulence intensity formulation for flat-plate boundary layers. Phys. Fluids 15, 2461-2464.

Marusic, I., McKeon, B.J., Monkewitz, P.A., Nagib, H.M., Smits, A.J. \& Sreenivasan, K.R. 2010 Wall-bounded turbulent flows at high Reynolds numbers: recent advances and key issues. Phys. Fluids 22, 065103.

Marusic, I. \& Monty, J.P. 2019 Attached eddy model of wall turbulence. Annu. Rev. Fluid Mech. 51 (1), 49-74.

Marusic, I., Monty, J.P., Hultmark, M. \& Smits, A.J. 2013 On the logarithmic region in wall turbulence. J. Fluid Mech. 716, R3.

Metzger, M.M. \& Klewicki, J.C. 2001 A comparative study of near-wall turbulence in high and low Reynolds number boundary layers. Phys. Fluids 13 (3), 692-701.

Monkewitz, P.A. 2021 The late start of the mean velocity overlap log law at $y^{+}=O\left(10^{3}\right)$ - a generic feature of turbulent wall layers in ducts. J. Fluid Mech. 910, A45.

Monkewitz, P.A. \& NagiB, H.M. 2015 Large Reynolds number asymptotics of the stream-wise normal stress in ZPG turbulent boundary layers. J. Fluid Mech. 783, 474-503.

Monkewitz, P.A., Nagib, H.M. \& Boulanger, V. 2017 Comparing the three possible scalings of stream-wise normal stress in turbulent boundary layers. In Proceedings of TSFP10, Chicago (USA) (ed. A.J. Smits \& H.M. Nagib). www.tsfp-conference.org.

Morrison, J.F., McKeon, B.J., JiAng, W. \& Smits, A.J. 2004 Scaling of the streamwise velocity component in turbulent pipe flow. J. Fluid Mech. 508, 99-131.

MusKer, A.J. 1979 Explicit expression for the smooth wall velocity distribution in a turbulent boundary layer. AIAA J. 17, 655-657.

NaGiB, H.M. \& CHAUhan, K.A. 2008 Variations of von Kármán coefficient in canonical flows. Phys. Fluids 20, 101518 .

Perry, A.E., Henbest, S.M. \& CHOng, M.S. 1986 A theoretical and experimental study of wall turbulence. J. Fluid Mech. 165, 163-199.

Pirozzoli, S., Romero, J., Fatica, M., Verzicco, R. \& Orlandi, P. 2021 One-point statistics for turbulent pipe flow up to $r e_{\tau} \approx 6000$. J. Fluid Mech. 926, A28.

Pope, S.B. 2000 Turbulent Flows. Cambridge University Press.

Pullin, D.I., InOue, M. \& SAito, N. 2013 On the asymptotic state of high Reynolds number, smooth-wall turbulent flows. Phys. Fluids 25, 015116. 


\section{Streamwise Reynolds stress}

Samie, M., Marusic, I., Hutchins, N., Fu, M.K., Fan, Y., Hultmark, M. \& Smits, A.J. 2018 Fully resolved measurements of turbulent boundary layer flows up to $R e_{\tau}=20000$. J. Fluid Mech. 851, 391-415.

Sillero, J.A., JiméneZ, J. \& Moser, R.D. 2013 One-point statistics for turbulent wall-bounded flows at Reynolds numbers up to $\delta^{+} \approx 2000$. Phys. Fluids 25, 105102.

Smits, A.J., Hultmark, M., Lee, M., Pirozzoli, S. \& Wu, X. 2021 Reynolds stress scaling in the near-wall region of wall-bounded flows. J. Fluid Mech. 926, A31.

Smits, A.J., McKeon, B.J. \& Marusic, I. 2011 High Reynolds number wall turbulence. Annu. Rev. Fluid Mech. 43 (1), 353-375.

SPAlART, P.R. \& ABE, H. 2021 Empirical scaling laws for wall-bounded turbulence deduced from direct numerical simulations. Phys. Rev. Fluids 6, 044604.

Thais, L., Mompean, G. \& GATSKI, T. 2013 Spectral analysis of turbulent viscoelastic and newtonian channel flows. J. Non-Newtonian Fluid Mech. 200, 165-176.

Townsend, A.A. 1976 The Structure of Turbulent Shear Flow. Cambridge University Press.

Vallikivi, M., Hultmark, M. \& Smits, A.J. 2015 Turbulent boundary layer statistics at very high Reynolds number. J. Fluid Mech. 779, 371-389.

VAllikivi, M. \& SMits, A.J. 2014 Fabrication and characterization of a novel nanoscale thermal anemometry probe. IEEE J. Microelectromech. Syst. 23, 899-907.

WILCOX, D.C. 1995 Perturbation Methods in the Computer Age. DCW Industries. 